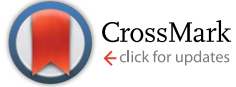

Cite this: Soft Matter, 2015, 11, 2906

Received 1st December 2014 Accepted 2nd March 2015

DOI: $10.1039 / c 4 s m 02646 b$

\section{Proteo-lipobeads for the oriented encapsulation of membrane proteins $\uparrow$}

\author{
Pinar Frank, $t^{a}$ Bernhard Siebenhofer, $\neq^{a}$ Theresa Hanzer, $t^{a}$ Andreas F. Geiss, ${ }^{a}$ \\ Florian Schadauer, ${ }^{a}$ Ciril Reiner-Rozman, ${ }^{a}$ Bill Durham, ${ }^{b}$ Leslie M. Loew, $^{c}$ \\ Bernd Ludwig, ${ }^{d}$ Oliver-M. H. Richter, ${ }^{d}$ Christoph Nowak ${ }^{e}$ and Renate L. C. Naumann ${ }^{\star a}$
}

www.rsc.org/softmatter

As a surrogate of live cells, proteo-lipobeads are presented, encapsulating functional membrane proteins in a strict orientation into a lipid bilayer. Assays can be performed just as on live cells, for example using fluorescence measurements. As a proof of concept, we have demonstrated proton transport through cytochrome $c$ oxidase.

Membrane proteins (MPs) are the target of about $60 \%$ of all pharmaceuticals. ${ }^{1}$ Therefore, there is great interest in bioassays for drug screening purposes of these proteins. To assay ion transport properties, MPs need to be present within lipid bilayers. Such bioassays are mostly performed on live cells. ${ }^{2}$ Proteoliposomes can be used as an alternative, ${ }^{3}$ which are unfortunately not comparable to live cells regarding their stability or the control over the orientation of the MPs. The issue of stability was solved successfully by the development of polymerosomes, ${ }^{4}$ which do not permit the oriented encapsulation of MPs. A uniform orientation of MPs is a necessary

${ }^{a}$ Biosensor Technologies, Austrian Institute of Technology GmbH, AIT, Donau-City Str 1, 1220 Vienna, Austria.E-mail: Renate.Naumann@ait.ac.at

${ }^{b}$ Department of Chemistry, University of Arkansas, Fayetteville, AR 72701, USA

${ }^{c} R$. D. Berlin Center for Cell Analysis and Modeling, University of Connecticut Health Center, Farmington, Connecticut 06030, USA

${ }^{d}$ Biocenter, Goethe University, Inst. of Biochemistry, Max-von-Laue-Str. 9, D-60438, Frankfurt, Germany

${ }^{e}$ Center of Electrochemical Surface Technology, CEST, Viktor-Kaplan-Straße 2, 2700 Wiener Neustadt, Austria

$\dagger$ Electronic supplementary information (ESI) available: Formal names and excitation/emission wavelengths of fluorescent labels. Details of the preparation protocol and additional LSM images demonstrating the polydispersity and stability of the PLBs. See DOI: 10.1039/c4sm02646b

\$ Pinar Frank, Bernhard Siebenhofer and Theresa Hanzer contributed equally to this work. The research was supported under the Austrian Federal Ministry for Transport, Innovation and Technology (GZ BMVIT-612.166/0001-III/I1/2010) and the City of Vienna (MA 7). Partial support of this work was provided by ZIT, Center of Innovation and Technology of Vienna. L. M. Loew acknowledges support by NIH grant R01 EB001963. B. Durham acknowledges support by NIH grant P30 GM103450. We acknowledge the assistance of Prof. Michael Boersch, University of Jena, Germany, in the field of LSM. Proteo-lipobeads: European Patent pending, Nr. 12198401.7. prerequisite for bioassays, particularly regarding ion transport through ion channels and transporters.

We have therefore developed proteo-lipobeads (PLBs), a biomimetic system for the oriented encapsulation of MPs in a functionally active form. PLBs are based on micrometer sized agarose beads modified with small linker molecules such as NTA (nitrilotriacetic acid) terminated $\mathrm{CH}_{2}$ chains. The MPs are bound to these chains via histidine(his)-tags, a well-established method for the oriented immobilization of MPs. ${ }^{7}$ After immobilization of the MPs, these particles are subjected to dialysis in the presence of solubilized phospholipids. Thereby, MPs are reconstituted into bilayer lipid membranes (BLMs) to form PLBs. The final structure is depicted schematically in Scheme 1. Similar systems have been presented before. For instance, a seven transmembrane segment protein as well as the proximal lipid layer have been attached to paramagnetic beads using antibody technology. Proteoliposomes were then formed by dialysis with lipid micelles. ${ }^{5}$ This format, however, was designed for binding assays whereas functional assays were not presented. To show the capability of PLBs as functional assays we use cytochrome $c$ oxidase $(\mathrm{C} c \mathrm{O})$ from $P$. denitrificans with the his-tag attached to subunit (SU) I.

The reconstitution of MPs into a BLM, thereby using the immobilized proteins as a scaffold, has been investigated previously on flat surfaces. ${ }^{8,9} \mathrm{C} c \mathrm{O}$ from $R$. sphaeroides with the his-tag attached to SU II was immobilized in the reverse orientation compared to the one used in the present study. A small aqueous layer between the surface and lipid layer was indicated indirectly by electrochemical detection of protons pumped through the $\mathrm{C} c \mathrm{O}$ in the direction of the surface. An aqueous layer on both sides of the BLM is a further prerequisite for functional assays. The agarose gel used to form PLBs can be considered to provide an additional reservoir for water and ions, as shown by the accumulation of the water-soluble fluorescent dye SNARF-1, even in the presence of the lipid bilayer (Fig. S2 and Table S1 $\dagger$ ). Hence the PLBs can be expected to provide a format not only for binding but also for functional assays. Changes of physical parameters within or in close 


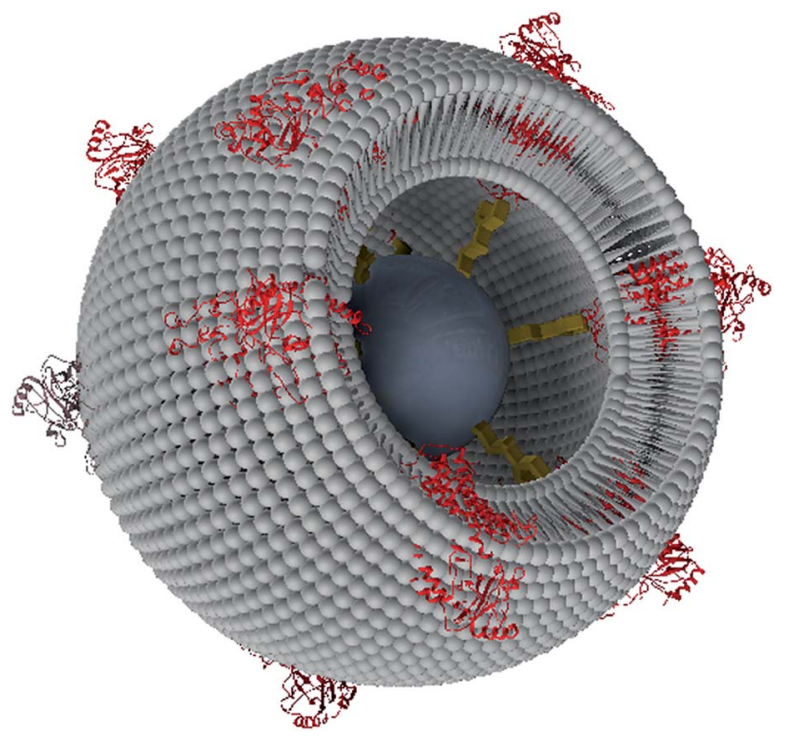

Scheme 1 Schematic of a proteo-lipobead (PLB) based on an agarose bead (grey) modified by an NTA-terminated linker (yellow) with $\mathrm{CcO}$ (red) immobilized in strict orientation via a his-tag attached to the cytoplasmically oriented C-terminus of SU I. A DiphyPC (diphytanoylsn-glycero-3-phosphocholine) bilayer (grey) is formed between the proteins during dialysis. The layered structure is not drawn true to scale.

proximity to the protein/membrane system can be monitored as a function of time using laser scanning confocal microscopy (LSM). ${ }^{6}$

We used LSM to visualize the lipid layer of the PLBs, employing fluorescent labeled lipids such as NBD-PE as well as the potential sensitive dyes (PSDs), di-8-ANEPPS and di-4ANBDQBS (red-shifted PSD) (Fig. 1). Formal names and excitation/emission wavelengths are given in ESI Table S1. $\dagger$ Fig. 1c and $\mathrm{d}$ show the polydispersity of the PLBs ranging from 50-150 $\mu \mathrm{m}$.

Hemicyanine-based membrane probes like di-8-ANEPPS and di-4-ANBDQBS exhibit negligible fluorescence in aqueous solution but fluoresce strongly when bound to BLMs. ${ }^{10}$ Fig. 1a illustrates a BLM layer of regular fluorescence emission intensity around the bead. BLMs are known to assemble on silica beads by themselves. ${ }^{11}$ Therefore, as a control experiment, gel beads were subjected to dialysis in the absence of immobilized $\mathrm{CcO}$. Fluorescence emission can be seen in the presence of di-4-ANBDQBS, however, broader and more blurry than the BLM in the presence of immobilized MPs (Fig. 1b). All of the images discussed so far were recorded in the equatorial plane of the beads (Fig. S3a†). Laser scanning images with the focal plane adjusted to the top of the PLB showed a continuous fluorescence of smaller diameter as expected for a BLM fully enclosing the beads (Fig. S3b †). We concluded from these results that using $\mathrm{C} c \mathrm{O}$ as a scaffold leads to well-defined lipid bilayers evenly covering the entire surface of the gel bead.

$\mathrm{CcO}$ immobilized with the his-tag attached to SU I is oriented with the cytochrome $c(\mathrm{ccc})$ binding site pointing to the outside of the membrane. A polyclonal primary Immunoglobulin G (IgG)antibody from rabbit specific to both SU I and II was bound to
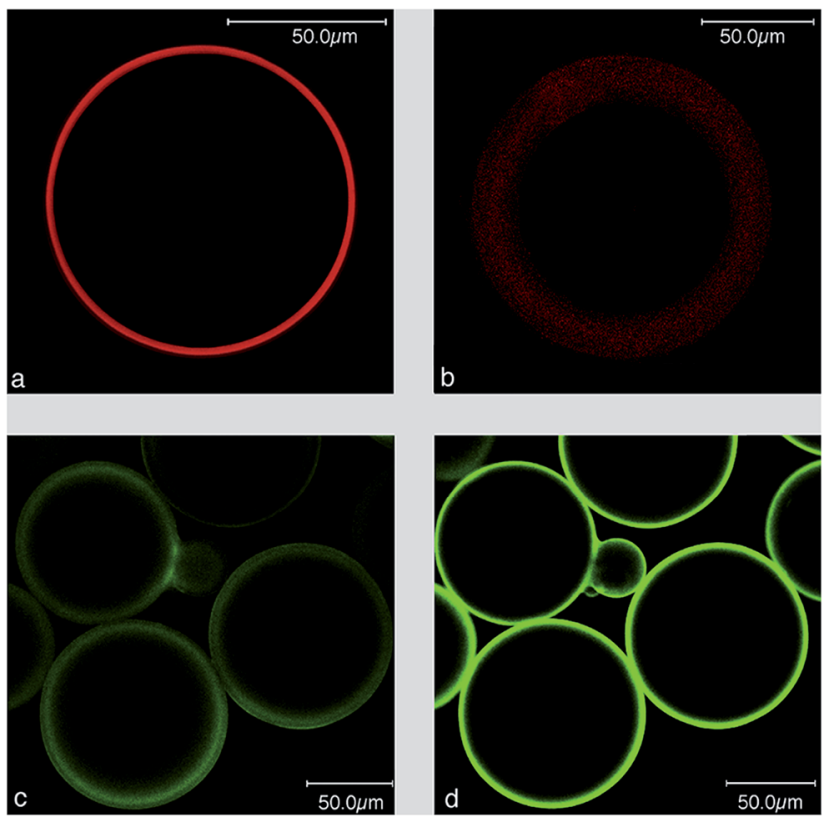

Fig. 1 Laser scanning images of PLBs labelled with NBD-PE, di-8ANEPPS or di-4-ANBDQBS (a) in the presence and (b) absence of $\mathrm{CcO}$. Laser scanning images taken at the equatorial plane of $\mathrm{CCO}$ based PLBs with a primary antibody specifically bound to SU I and SU II of $\mathrm{CcO}$. A Cy5 conjugated secondary antibody was used as a fluorescent label (c) in the absence and (d) presence of di-4-ANBDQBS in the lipid phase.

the PLBs. Subsequently, a Cy5 conjugated secondary antibody was bound to the primary IgG and the Cy5 label was excited by a $\mathrm{HeNe}$ laser at $633 \mathrm{~nm}$. The presence of $\mathrm{C} C \mathrm{O}$ was indicated by the fluorescence of the Cy5 label in the presence and absence of di4-ANBDQBS (Fig. 1c and d).

Finally, a functional assay was conducted considering that $\mathrm{CcO}$, as the terminal complex of the respiratory chain, converts the free enthalpy gained by the reduction of oxygen to water into a difference in electrochemical potentials of protons, $\Delta \tilde{\mu} H^{+}$ across the lipid membrane. $\Delta \tilde{\mu} H^{+}$consists of the membrane potential, $\Delta \Phi$, and a difference in $\mathrm{pH}$ values, $\Delta \mathrm{pH}$, between the inner and outer aqueous phases. In our case, the $\mathrm{c} c$ binding site is located at the outer side of the PLB. Proton transport can be initiated by light activation of photoactive electron donors bound to the $\mathrm{cc}$ binding site that inject electrons into the $\mathrm{CcO}$. In this work we used the $\mathrm{Ru}$ complex $\mathrm{Ru}_{2} \mathrm{C}\left(\left[(\mathrm{bpy})_{2} \mathrm{Ru}\right.\right.$ (diphen) $\left.\left.\mathrm{Ru}(\mathrm{bpy})_{2}\right]\left(\mathrm{PF}_{6}\right)_{4}\right)$ as an electron donor, mixed with aniline as a sacrificial electron donor and 3CP (3-carboxy-PROXYL) to prevent proton release from aniline. ${ }^{12} \mathrm{pH}$ changes at the outside of the PLB can be conveniently detected by fluorescein DHPE (Table S1 $\dagger$ ), a sensor molecule that incorporates into the distal leaflet of the lipid bilayer under the conditions described in the ESI. $\dagger$ A decrease of $\mathrm{pH}$ can be detected by a decrease of fluorescence intensity. LSM images and fluorescence intensities were recorded within localized areas before and after continuous illumination with a halogen lamp in the presence and absence of $\mathrm{Ru}_{2} \mathrm{C}, 3 \mathrm{CP}$ and aniline (Fig. S5 $\dagger$ ). As shown in Fig. 2, fluorescence intensities decrease in a time scale of seconds by $10 \%$ and $42 \%$ in the buffered and unbuffered $\mathrm{KCl}$ solution, 
a)

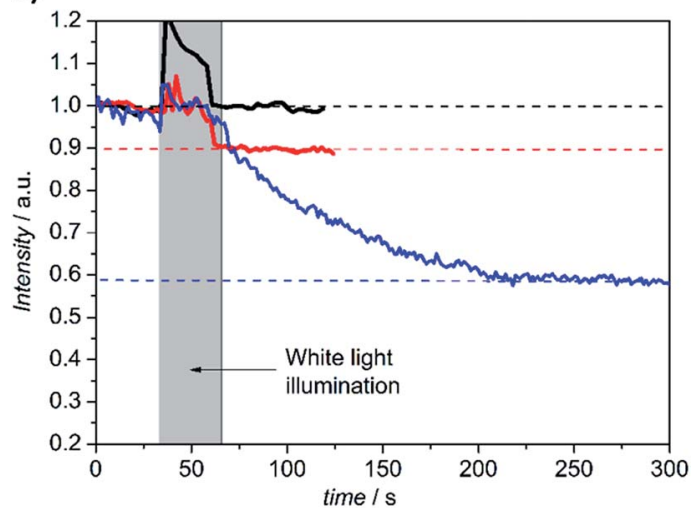

b)

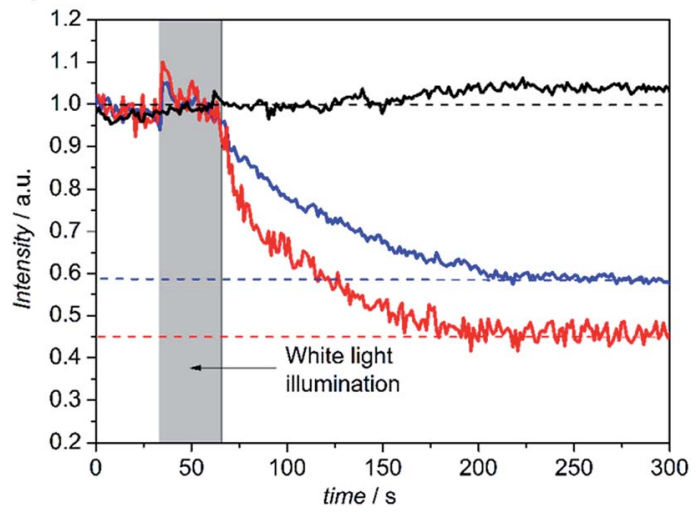

Fig. 2 (a) Time dependent change of the relative fluorescence intensity of membrane bound fluorescein DHPE before and after continuous illumination with a halogen lamp with Ru2C, 3CP and aniline in $40 \mathrm{mM} \mathrm{KCl}$ solution (blue) compared to the same PLBs with (red) and without (black) Ru2C, 3CP and aniline in Tris- $\mathrm{HCl} / \mathrm{KCl}(5 \mathrm{mM} /$ $35 \mathrm{mM}$ ) buffer. (b) Time dependent change of the relative fluorescence intensity of membrane bound fluorescein DHPE before and after continuous illumination with a halogen lamp with Ru2C, 3CP and aniline in $40 \mathrm{mM} \mathrm{KCl}$ solution (blue) in the presence of valinomycin alone (red) and valinomycin and FCCP (black).

respectively. This effect was abolished under uncoupling conditions in the presence of valinomycin and FCCP (carbonyl cyanide 4-(trifluoromethoxy)phenylhydrazone), which render the membrane permeable to potassium ions and protons, respectively, whereas in the presence of valinomycin alone the intensity dropped by $55 \%$ due to the collapse of the membrane potential.

This behaviour is characteristic for an active transporter strongly affected by the membrane potential. Time-resolved measurements of proton release have been reported, in the time scale of $\mu$ s to $\mathrm{ms}$, using $\mathrm{CcO}$ reconstituted in liposomes. ${ }^{12,13}$ These results, however, are not directly comparable with our work, because single laser flashes were used for excitation. Moreover, further work reported in a separate publication leads us to believe that the turnover rate is changed in PLBs. Note, in this context, that fluorescein DHPE monitors protons in the immediate vicinity of the lipid bilayer, rather than the aqueous phase as in ref. 13. This will be investigated in more detail in a separate publication.

\section{Conclusions}

Our experiments demonstrate the applicability of PLBs as a new platform for functional assays of membrane proteins. Further work was done using PLBs ranging in size down to the $\mathrm{nm}$ scale. ${ }^{14}$ The advantages of the new system over lipid vesicles are the uniform orientation of the incorporated proteins combined with the considerably increased stability. LSM assays shown here may be transferred easily to other formats, such as fluorescence-activated cell sorting (FACS) or fluorescence imaging plate reader (FLIPR), which are more feasible for drug screening purposes than LSM.

\section{Notes and references}

1 M. A. Yildirim, K.-I. Goh, M. E. Cusick, A.-L. Barabasi and M. Vidal, Nat. Biotechnol., 2007, 25, 1119-1126.

2 J. E. Gonzalez, K. Oades, Y. Leychkis, A. Harootunian and P. A. Negulescu, Drug Discovery Today, 1999, 4, 431-439.

3 G. Nordlund, J. B. S. Ng, L. Bergstrom and P. Brzezinski, ACS Nano, 2009, 3, 2639-2646.

4 S. May, M. Andreasson-Ochsner, Z. Fu, Y. X. Low, D. Tan, H.-P. M. de Hoog, S. Ritz, M. Nallani and E.-K. Sinner, Angew. Chem., Int. Ed., 2013, 52, 749-753.

5 T. Mirzabekov, H. Kontos, M. Farzan, W. Marasco and J. Sodroski, Nat. Biotechnol., 2000, 18, 649-654.

6 N. S. Claxton, T. J. Fellers and M. W. Davidson, Laser Scanning Confocal Microscopy, Bios Scientific Publishers, Oxford, 1987.

7 S. A. Trammell, L. Y. Wang, J. M. Zullo, R. Shashidhar and N. Lebedev, Biosens. Bioelectron., 2004, 19, 1649-1655.

8 F. Giess, M. G. Friedrich, J. Heberle, R. L. Naumann and W. Knoll, Biophys. J., 2004, 87, 3213-3220.

9 A. Kibrom, R. F. Roskamp, U. Jonas, B. Menges, W. Knoll, H. Paulsen and R. L. C. Naumann, Soft Matter, 2011, 7, 237-246.

10 E. Fluhler, V. G. Burnham and L. M. Loew, Biochemistry, 1985, 24, 5749-5755.

11 E. Reimhult, M. Zach, F. Hook and B. Kasemo, Langmuir, 2006, 22, 3313-3319.

12 I. Belevich, D. A. Bloch, N. Belevich, M. Wikstrom and M. I. Verkhovsky, Proc. Natl. Acad. Sci. U. S. A., 2007, 104, 2685-2690.

13 K. Kirchberg, H. Michel and U. Alexiev, J. Biol. Chem., 2012, 287, 8187-8193.

14 P. Frank, B. Siebenhofer, T. Hanzer, A. F. Geiss, F. Schadauer, C. Reiner-Rozman, B. Durham, L. M. Loew, B. Ludwig, O.-M. H. Richter, C. Nowak and R. L. C. Naumann, Soft Matter, 2015, DOI: 10.1039/ C4SM02646B. 